Interfaces and Free Boundaries 13 (2011), 411-421

DOI $10.4171 / \mathrm{IFB} / 265$

\title{
Pinning of interfaces in random media
}

\author{
NICOLAS DIRR \\ Cardiff School of Mathematics, Cardiff University, \\ Senghennydd Road, Cardiff, Wales, UK, CF24 4AG \\ E-mail:DirrNP@cardiff.ac.uk \\ PATRICK W. DONDL \\ Institut für Angewandte Mathematik, Universität Bonn, \\ Endenicher Allee 60, D-53115 Bonn, Germany \\ E-mail:pwd@hcm.uni-bonn.de \\ MichAEL SCHEUTZOW \\ Fakultät II, Institut für Mathematik, Sekr. MA 7-5, Technische Universität Berlin, \\ Strasse des 17. Juni 136, D-10623 Berlin, Germany \\ E-mail:ms@math.tu-berlin.de
}

[Received 21 September 2010 and in revised form 24 June 2011]

\begin{abstract}
For a model for the propagation of a curvature sensitive interface in a time independent random medium, as well as for a linearized version which is commonly referred to as Quenched EdwardsWilkinson equation, we prove existence of a stationary positive supersolution at non-vanishing applied load. This leads to the emergence of a hysteresis that does not vanish for slow loading, even though the local evolution law is viscous (in particular, the velocity of the interface in the model is linear in the driving force).
\end{abstract}

2010 Mathematics Subject Classification: Primary 35R60; Secondary 74N20.

Keywords: QEW; phase boundaries; pinning; random environment.

\section{Introduction}

Problems of interface evolution in heterogeneous media arise in a large number of physical models. Common to such models is a regularizing operator, for example line tension, and the competition between an external applied driving force $F$ and a force field $f(x, y)$ describing the inhomogeneities. Assuming a viscous law for the relation between the driving force and the velocity of the interface, an important question is whether rate independent hysteresis can emerge in an average sense from the interaction between the heterogeneous force field and the regularizing operator.

In this article, we consider a model for the evolution of an interface driven by its mean curvature through a random field of obstacles. Let $n \in \mathbb{N}, n \geqslant 1$. Let $(\Omega, \mathcal{F}, \mathbf{P})$ be a probability space, $\omega \in \Omega$. We model the interface as the graph $(x, u(x, t, \omega))$ of a function $u: \mathbb{R}^{n} \times \mathbb{R} \times \Omega \rightarrow \mathbb{R}$ moving through a field $f(x, y, \omega)$ of (soft) random obstacles and a constant driving force $F$. More 
precisely, we consider the PDE

$$
\begin{aligned}
\partial_{t} u(x, t, \omega)= & \sqrt{1+|\nabla u(x, t, \omega)|^{2}} \operatorname{div}\left(\frac{\nabla u(x, t, \omega)}{n \sqrt{1+|\nabla u(x, t, \omega)|^{2}}}\right) \\
& +\sqrt{1+|\nabla u(x, t, \omega)|^{2}}(f(x, u(x, t, \omega))+F) \\
= & : \sqrt{1+|\nabla u(x, t, \omega)|^{2}}(\kappa(u(x, t, \omega))+f(x, u(x, t, \omega))+F), \\
u(x, 0, \omega)= & 0 .
\end{aligned}
$$

The first term on the right hand side of equation (1.1) is the mean curvature operator for a surface that is given as the graph of the function $u$. The second term is the driving force, split up into the non-homogeneous random part $f$ and the external constant loading $F$. The random field $f$ will be specified in Section 2 in Conditions 2.1 and 2.2. Basically we will assume that the nonhomogeneity consists of randomly distributed individual obstacles of a fixed smooth shape and possibly random strength. By $\kappa(u(x, t, \omega))$ we denote the mean curvature operator for the graph of a function $u(\cdot, t, \omega)$ evaluated at $x$.

Equation (1.1) is motivated in the following way (see also [CD]): A very basic model for an interface (phase boundary, dislocation line in its slip plane etc.) moving through an array of random obstacles (e.g. impurities, other dislocation lines) in an over-damped limit (inertial effects are neglected) is the gradient flow of the area functional plus a random bulk term. Consider thus a bounded set $U \subset \mathbb{R}^{n+1}$ and a smooth hypersurface $\Gamma$ that is the boundary of the set $A_{\Gamma} \subset U$ and define the energy

$$
E(\Gamma):=\mathcal{H}^{1}(\Gamma)+\int_{A_{\Gamma}}(f(X, \omega)+F) \mathrm{d} X .
$$

Here, $\mathcal{H}^{1}$ denotes the 1-dimensional Hausdorff measure. The first inner variation (i.e., deforming the interface with the flow of a smooth vector field) yields the mean curvature $\kappa$ and a bulk term. The viscous gradient flow with respect to the energy (1.3) is thus given by the evolution equation

$$
v_{n}(x)=\kappa(x)+f(x, \omega)+F, \quad x \in \Gamma,
$$

for the normal velocity $v_{n}$ of the interface. Now it is also possible to extend the notion of an evolving interface to $U=\mathbb{R}^{n+1}$. The model is called quenched, because the random field does not explicitly depend on time. For forced mean curvature flow and applications, in particular in the case of periodic forcing, we refer to [CL, CB, DK]. Since we are only interested in constructing a supersolution for (1.4) when the initial surface is flat, it is sufficient to consider the mean curvature flow for an interface that is the graph of a function $u$, i.e., $\Gamma=\left\{(x, y): y=u(x), x \in \mathbb{R}^{n}\right\}$.

If the gradient of $u$ is sufficiently small, the evolution by forced mean curvature flow (MCF) for the graph can be approximated heuristically by a semilinear parabolic PDE of the form

$$
\begin{aligned}
\partial_{t} u(x, t, \omega) & =\Delta u(x, t, \omega)+f(x, u(x, t, \omega), \omega)+F \quad \text { on } \mathbb{R}^{n}, \\
u(x, 0, \omega) & =0 .
\end{aligned}
$$

These kinds of problems have found considerable interest in the physics community (see e.g. [Ka, $\mathrm{Kl}, \mathrm{B}]$ ). They are often referred to as the Quenched Edwards-Wilkinson (QEW) model.

The goal of this article is to construct, for some $F>0$, a stationary supersolution $v$ to (1.1) and to (1.5) satisfying $v \geqslant 0$. In this article, we consider the natural case where, due to the randomness 
of the obstacle field, there exist arbitrarily large simply connected domains with positive combined driving force (i.e., where $f+F>0$ ), in other words, large areas without obstacle. This makes a purely analytical approach, as employed in [DY, DK], insufficient.

In order to illustrate the difficulty, consider in $1+1$ dimensions a periodic array of obstacles with a forcing $F>0$. Now remove each obstacle independently with rate $p \ll 1$ and increase the obstacle strength by $\delta(p)$ in order to keep the expected value of the obstacle strength equal to the periodic one. The resulting random configuration (periodic with random "holes") may have a solution which is unbounded as $t \rightarrow \infty$ : For any $h \in \mathbb{Z}$ and $l \in \mathbb{N}$, there exists almost surely a $z \in \mathbb{Z}$ such that the ball $B_{l}((z, h))$ of radius $l$ centered at $(z, h)$ is free of obstacles. For sufficiently large $l$, the solution in this ball will grow to a height that is larger than $h+1$. For an appropriate choice of obstacles and parameters, this perturbation can grow like a kink-antikink pair in a reaction-diffusion equation (e.g. Allen-Cahn) with forcing, until the entire curve has moved up at least one unit. Then the process repeats itself at a "hole" at height level $h+1$.

If such a supersolution exists, by the comparison principle for the mean curvature flow and for parabolic equations, an evolving solution $u$ with any initial condition below $v$ will always remain below $v$-the interface is pinned. Such pinning of an interface leads to a hysteresis that does not vanish for slow loading in the physical system. To see this, consider a loading cycle starting with $F=0$, increasing at first. The interface remains pinned until the driving force reaches a critical value (see Section 3 for a brief discussion of depinning). Above the critical force the material transforms (switching polarization, for example). Upon reversal of the driving force, the same phenomenon occurs. ${ }^{1}$ One can see that plotting the transformed region vs. the driving force will show a hysteresis loop that does not vanish even for slowly varying driving force $F$.

In the following section, we construct such a supersolution under suitable assumptions on the random obstacle field. Section 3 provides some outlook.

\section{Construction of a supersolution}

We first pose two conditions that will fix the structure of the nonhomogeneous term $f$ in equations (1.1) and (1.5). This random nonlinearity $f$ is constructed in the following way: We consider an obstacle function $\phi \in C^{\infty}\left(\mathbb{R}^{n} \times \mathbb{R}\right)$ with the following properties:

Condition 2.1 (Obstacle shape) There exist $r_{1}, r_{0}$ with $r_{1}>\sqrt{n} r_{0}>0$ such that

(i) $\phi \leqslant 0, \phi(x, y)=0$ for $\|(x, y)\|>r_{1}$,

(ii) $\phi(x, y) \leqslant-1$ for $\|(x, y)\|_{\infty} \leqslant r_{0}$.

This fixes a "shape" for the individual obstacles. Here, $\|\cdot\|$ denotes the Euclidean norm on $\mathbb{R}^{n+1}$, and $\|\cdot\|_{\infty}$ denotes the maximum norm. The heterogeneity $f$ is now given as the sum over individual obstacles with centers $\left\{\left(x_{i}(\omega), y_{i}(\omega)\right)\right\}_{i \in \mathbb{N}}$ and strength $f_{i}(\omega) \geqslant 0$, i.e.,

$$
f(x, y, \omega)=\sum_{i} f_{i}(\omega) \phi\left(x-x_{i}(\omega), y-y_{i}(\omega)\right)
$$

\footnotetext{
1 There is a difficulty in modeling this behavior. In a physical situation, the obstacles (nontransforming inclusions, for example) will always obstruct the evolution of an interface moving in any direction. A reasonable way to express this would be to consider the driving force $f(x, u(x, t, \omega), \omega) \cdot \operatorname{sign}\left(\partial_{t} u\right)$. Such an additional nonlinearity in the equation would make the analysis unnecessarily complicated-we thus restrict ourselves to treating the transformation $\left(\partial_{t} u \geqslant 0\right)$ and the back transformation $\left(\partial_{t} u \leqslant 0\right)$ separately.
} 
We now pose a condition on the distribution of obstacles. The condition basically states that there is a uniform lower bound for finding an obstacle of some strength (also bounded from below) in a box of volume 1, independent of its shape or position, and independently for pairwise disjoint boxes.

CONDITION 2.2 (Obstacle distribution) The random distribution of obstacle sites $\left\{\left(x_{k}, y_{k}\right)\right\}_{k \in \mathbb{N}} \subset$ $\mathbb{R}^{n} \times\left[r_{1}, \infty\right)$ and strength $\left\{f_{k}\right\}_{k \in \mathbb{N}} \subset[0, \infty)$ satisfies

(i) $\left(x_{k}, y_{k}\right)$ are distributed according to an $n+1$-dimensional Poisson process on $\mathbb{R}^{n} \times\left[r_{1}, \infty\right)$ with intensity $\lambda>0$,

(ii) $f_{k}$ are iid strictly positive random variables which are independent of $\left\{x_{k}, y_{k}\right\}$.

Note that there are no obstacles crossing the line $\{y=0\}$, so at $t=0$ the interface with initial condition (1.2) only sees the external driving force. For a small time, the velocity of the interface is thus uniformly positive for $F>0$. The comparison principle ensures that we thus have $\partial_{t} u \geqslant 0$ for all times. To see this, assume that for a smooth solution to (1.5) there exists a first time $t_{0}>0$ when there exists $x_{0} \in \mathbb{R}^{n}$ with $\partial_{t} u\left(x_{0}, t_{0}\right)=0$. Differentiating (1.5) with respect to time yields

$$
\partial_{t t} u\left(x_{0}, t_{0}\right)=\Delta u_{t}\left(x_{0}, t_{0}\right)+f_{u}\left(x_{0}, u\left(x_{0}, t_{0}\right)\right) \partial_{t} u\left(x_{0}, t_{0}\right)=\Delta u_{t}\left(x_{0}, t_{0}\right) \geqslant 0 .
$$

Noting that, for a smooth solution of (1.1), the spatial gradient of $\partial_{t} u$ also vanishes at $\left(x_{0}, t_{0}\right)$, one can obtain the same nonnegativity result for the mean curvature flow.

As mentioned in the introduction, the main difficulty in this work stems from the fact that there exist, with positive probability, arbitrarily large areas with arbitrarily small obstacles. The strategy for the proof of pinning is thus as follows. We first divide our domain $\mathbb{R}^{n} \times \mathbb{R}$ into boxes of a certain side length and height, indexed by $\mathbb{Z}^{n} \times \mathbb{Z}$. We call a site "open" or "good" if it contains a suitably large obstacle. It is then necessary that good sites form a connected surface of a certain regularity: There should exist a (discrete) Lipschitz function $L: \mathbb{Z}^{n} \rightarrow \mathbb{Z}$ whose graph only consists of good sites. This percolation result is proved in [DD]; we repeat it in the paragraph below for the reader's convenience. See also [G] for an improved estimate on the critical percolation threshold. In the proof of Theorems 2.4 and 2.13 we can then use this cluster and the Lipschitz estimate to construct a supersolution using analytical tools.

ThEOREM 2.3 (Dirr-Dondl-Grimmett-Holroyd-Scheutzow) Let $n \geqslant 1$ and $p \in(0,1)$. We designate $z \in \mathbb{Z}^{n+1}$ open with probability $p$, and otherwise closed, with different sites receiving independent states. The corresponding probability measure on the sample space $\Omega=\{0,1\}^{\mathbb{Z}^{n+1}}$ is denoted by $\mathbf{P}_{p}$. We write $\|\cdot\|_{1}$ for the 1-norm on $\mathbb{Z}^{n+1}$. The following holds:

For any $n \geqslant 1$, if $p>1-(2 n+2)^{-2}=$ : $p_{c}$ then there exists a.s. a (random) function $L: \mathbb{Z}^{n} \rightarrow \mathbb{N}$ with the following properties:

(i) for each $x \in \mathbb{Z}^{n}$, the site $(x, L(x)) \in \mathbb{Z}^{n+1}$ is open,

(ii) for any $x, y \in \mathbb{Z}^{n}$ with $\|x-y\|_{1}=1$ we have $|L(x)-L(y)| \leqslant 1$,

(iii) for any isometry $\theta$ of $\mathbb{Z}^{n}$ the functions $L$ and $L \circ \theta$ have the same laws, and the random field $\left(L(x): x \in \mathbb{Z}^{n}\right)$ is ergodic under each translation of $\mathbb{Z}^{n}$,

(iv) there exists $A=A(p, d)<\infty$ such that

$$
\mathbf{P}_{p}(L(0)>k) \leqslant A v^{k}, \quad k \geqslant 0,
$$

where $v=(2 n+2)(1-p)<1$. 
We first prove the existence of a stationary positive supersolution to the semilinear equation (1.5), since the calculations are somewhat simpler.

THEOREM 2.4 (Existence of a pinned solution for QEW) If Conditions 2.1 and 2.2 are satisfied, then there exists $F^{*}>0$ and a nonnegative $v: \mathbb{R}^{n} \times \Omega \rightarrow[0, \infty)$ such that $0 \geqslant \Delta v(x, \omega)+$ $f(x, v(x, \omega), \omega)+F^{*}$ a.s., i.e., any solution to (1.5) with initial condition (1.6) and $F \leqslant F^{*}$ gets pinned.

The proof consists of a piecewise construction of the supersolution, so it is first necessary to give some estimates on the components that will be used. We denote by $B_{r}$ the open unit ball of radius $r$ around 0 .

DEFINITION 2.5 (Local solution) Given $r_{\text {out }}>r_{\text {in }}>0, F_{\text {in }}>0$, and $F_{\text {out }}<0$, let $v_{\text {in }}$ be the unique solution of $\Delta v_{\text {in }}=F_{\text {in }}$ on $B_{r_{\text {in }}} \subset \mathbb{R}^{n}, v_{\text {in }}=0$ on $\partial B_{r_{\text {in }}}$. Let $v_{\text {out }}$ be the unique solution of $\Delta v_{\text {out }}=F_{\text {out }}$ on $B_{r_{\text {out }}} \backslash B_{r_{\text {in }}} \subset \mathbb{R}^{n}$, with boundary conditions $v_{\text {out }}=0$ on $\partial B_{r_{\text {in }}}$ and $v \cdot \nabla v_{\text {out }}=0$ on $\partial B_{r_{\text {out }}}$. We define

$$
v_{\text {local }}:= \begin{cases}v_{\text {in }} & \text { on } B_{r_{\text {in }}}, \\ v_{\text {out }} & \text { on } B_{r_{\text {out }}} \backslash B_{r_{\text {in }}}, \\ \lim _{r \rightarrow r_{\text {out }}} v_{\text {out }}(r) & \text { on } B_{r_{\text {out }},} \\ \infty & \text { otherwise. }\end{cases}
$$

PROPOSITION 2.6 The function $v_{\text {local }}$ defined above satisfies:

(i) $v_{\text {local }}$ is radially strictly increasing on $B_{r_{\text {out }}}$,

(ii) the graph of $v_{\text {local }}$ restricted to $B_{r_{\text {in }}}$ is contained in the set $B_{r_{\text {in }}} \times\left[-\frac{F_{\text {in }}}{2 n} r_{\text {in }}^{2}, 0\right]$,

(iii) given $\bar{f}>0$, if

$$
F_{\text {in }} r_{\text {in }} \geqslant\left|F_{\text {out }}\right|\left(-r_{\text {in }}+\frac{r_{\text {out }}^{n}}{r_{\text {in }}^{n-1}}\right) \text {, }
$$

and if $\max \left\{r_{\mathrm{in}}, \frac{F_{\mathrm{in}}}{2 n} r_{\mathrm{in}}^{2}\right\} \leqslant r_{0}$, then $v_{\text {local }}$ satisfies, in the sense of distributions (and in the sense of viscosity solutions),

$$
0 \geqslant \Delta v_{\text {local }}+\bar{f} \phi\left(\cdot, v_{\text {local }}(\cdot)+r_{0}\right)+F \quad \text { on } B_{r_{\text {out }}}
$$

for all $F \leqslant \min \left\{-F_{\text {out }}, \bar{f}-F_{\text {in }}\right\}$.

Proof. The individual assertions are proved by a simple calculation.

(i) Follows immediately from the maximum principle.

(ii) The function $v_{\text {in }}$ is nothing but a parabola, namely $v_{\text {in }}(x)=\frac{F_{\text {in }}}{2 n}|x|^{2}-\frac{F_{\text {in }}}{2 n} r_{\text {in }}^{2}$. The assertion can be read off from this form.

(iii) From (ii) and from the assumption on $\phi$ in 2.1, one can see that for $x \in B_{r_{\text {in }}}$, we have $\phi\left(x, v_{\text {local }}(x)+r_{0}\right) \leqslant-1$. The property (2.2) for each individual piece of $v_{\text {local }}$ can then be seen directly from the definition of $v_{\text {local }}$. The assertion follows by noting that (2.1) implies that the first derivative jumps down going radially outward across $\partial B_{r_{\text {in }}} .{ }^{2}$ This implies that $v_{\text {local }}$ is the pointwise minimum of two supersolutions, thus a supersolution itself. The Laplacian is then a negative measure.

2 The term $\frac{F_{\text {in }}}{n} r_{\text {in }}$ is the radial derivative of $v_{\text {in }}$, and $-F_{\text {out }}\left(\frac{-r_{\text {in }}}{n}+\frac{r_{\text {out }}^{n}}{n r_{\text {in }}^{n-1}}\right)$ is the radial derivative of $v_{\text {out }}$, at $\partial B_{r_{\text {in }}}$. 
DEFINITION 2.7 (Rescaling) Given $l>2 r_{1}, d>0, h>0$, and $k=\left(k_{1}, \ldots, k_{n}\right) \in \mathbb{Z}^{n}, j \in \mathbb{N}$, let

$$
\begin{aligned}
\tilde{Q}_{k} & :=\prod_{i=1}^{n}\left[k_{i}(l+d)+r_{1}, k_{i}(l+d)+l-r_{1}\right], \\
\bar{Q}_{k} & :=\prod_{i=1}^{n}\left[k_{i}(l+d), k_{i}(l+d)+l\right], \\
\bar{Q} & :=\bigcup_{k} \bar{Q}_{k} \quad \text { and } \quad \bar{D}:=\mathbb{R}^{n} \backslash \bar{Q}, \\
\tilde{Q}_{k, j} & =\tilde{Q}_{k} \times\left[(j-1) h+r_{1}, j h+r_{1}\right] .
\end{aligned}
$$

Here $\prod_{i=1}^{n}$ denotes the cartesian product of the $n$ intervals following.

REMARK The sets $\bar{Q}$ and $\bar{D}$ split $\mathbb{R}^{n}$ into cubes, separated by a distance $d$. The reduced cubes $\tilde{Q}_{k}$ are smaller by the length $2 r_{1}$ in every dimension, so that an obstacle with center in $\tilde{Q}_{k}$ fits completely inside $\bar{Q}_{k}$. The sets $\tilde{Q}_{k, j}$ are extended in the $n+1$-st direction by a height $h$.

Proposition 2.8 (Percolating obstacles) Given $h>0$, fix $l(h)>0$ and $\bar{f}>0$ such that

$$
1-\exp \left\{-\lambda|A| \cdot \mathbf{P}\left\{f_{1} \geqslant \bar{f}\right\}\right\}>p_{c}
$$

for $|A|=\left(l-2 r_{1}\right)^{n} h$, i.e., $l(h)=C_{0}\left(p_{c}, \lambda, \mathbf{P}\left\{f_{1} \geqslant \bar{f}\right\}\right) h^{-1 / n}+2 r_{1}$. Then there exists a random function $L: \mathbb{Z}^{n} \rightarrow \mathbb{N}$ with Lipschitz constant 1 such that, a.s., for all $k \in \mathbb{Z}^{n}$, there exists $i \in \mathbb{N}$ such that $\left(x_{i}, y_{i}\right) \in \tilde{Q}_{k, L(k)}$ and $f_{i} \geqslant \bar{f}$.

For each $k \in \mathbb{Z}^{n}$ we select one obstacle index $i \in \mathbb{N}$ with the above property and collect these obstacle indices in the set $I$.

Proof. This is a direct consequence of Theorem 2.3. Indeed, considering a cuboid $\tilde{Q}_{k, j}$ to be open if it contains an obstacle of strength greater than or equal to $\bar{f}$, we find that a cuboid is open with probability greater than $p_{c}$.

DEFINITION 2.9 We define the flat supersolution $v_{\text {flat }}: \mathbb{R}^{n} \rightarrow \mathbb{R}$ by the formula $v_{\text {flat }}(x):=$ $\min _{i \in I} v_{\text {local }}\left(x-x_{i}\right)$.

Proposition 2.10 Fix $h>0, \bar{f}>0$, and $l(h)$ as in Proposition 2.8. Let $r_{\text {out }}=\sqrt{n}(l(h)+$ $\left.d / 2-r_{1}\right)$ and assume that $r_{\text {out }}, r_{\text {in }}, F_{\text {in }}$ and $F_{\text {out }}$ satisfy the conditions in Proposition 2.6. Then $v_{\text {flat }}$ satisfies, a.s., in the sense of distributions (and in the sense of viscosity solutions),

$$
0 \geqslant \Delta v_{\text {flat }}(x)+\sum_{i \in I} \bar{f} \phi\left(x-x_{i}, v_{\text {flat }}(x)+r_{0}\right)+F \quad \text { on } \mathbb{R}^{n}
$$

for all $F \leqslant \min \left\{-F_{\text {out }}, \bar{f}-F_{\text {in }}\right\}$.

Proof. Since $v_{\text {flat }}$ is a minimum over shifted copies of the function $v_{\text {local }}$, which is a supersolution where it is not equal to $+\infty$ as proved in Proposition 2.6, it is enough to show that $v_{\text {flat }}(x)<+\infty$ for all $x \in \mathbb{R}^{n}$. This is, however, true by the choice of $r_{\text {out }}=\sqrt{n}\left(l(h)+d / 2-r_{1}\right)$ with the property that the union over all $k \in \mathbb{Z}^{n}$ of closed balls of this radius with centers anywhere in $\tilde{Q}_{k}$ covers all of $\mathbb{R}^{n}$.

REMARK Since the function $v_{\text {local }}$ is strictly increasing on $B_{r_{\text {out }}}$, the minimization process assigns each obstacle center $x_{i}$ its Voronor cell. On the Voronor cell associated with $x_{i}$, the function $v_{\text {local }}$ centered at $x_{i}$ attains the minimum. 
Proposition 2.11 (Gluing function) Fix $h>0, d>0, l>0$. Let $L: \mathbb{Z}^{n} \rightarrow \mathbb{R}$ be a function with the property that if $x, y \in \mathbb{Z}^{n}$ with $\|x-y\|_{1}=1$ we have $|L(x)-L(y)| \leqslant 2 h$. Then there exists $C_{1}>0$, depending only on the dimension $n$, such that there exists a smooth function $v_{\text {glue }}: \mathbb{R}^{n} \rightarrow \mathbb{R}$ such that

(i) for all $k \in \mathbb{Z}^{n}, v_{\text {glue }}(x)=L(k)$ if $x \in \bar{Q}_{k}$,

(ii) $\operatorname{supp} \nabla v_{\text {glue }} \subset \bar{D}$,

(iii) $\left\|D^{2} v_{\text {glue }}\right\|_{\infty} \leqslant C_{1} h / d^{2}$,

(iv) $\left\|\nabla v_{\text {glue }}\right\|_{\infty} \leqslant C_{1} h / d$.

Proof. It suffices to take a piecewise constant function that changes values on the center hyperplanes of the set $\bar{D}$ and apply a standard mollifier of size $d / 2$.

We have now collected all the components to construct the supersolution.

Proof of Theorem 2.4. First, fix $\bar{f}$ and the function $l(h)$ as in Proposition 2.8. Then fix $0<F_{\text {in }}<$ $\bar{f} / 2$ and $r_{\text {in }}$ such that $\max \left\{r_{\text {in }}, \frac{F_{\text {in }}}{2 n} r_{\text {in }}^{2}\right\} \leqslant r_{0}$.

We now need to find $d>0$ and $h>0$ such that, with $r_{\text {out }}$ chosen as in Proposition 2.10,

$$
\begin{aligned}
F_{\text {in }} r_{\text {in }} & \geqslant\left|F_{\text {out }}\right|\left(-r_{\text {in }}+\frac{r_{\text {out }}^{n}}{r_{\text {in }}^{n-1}}\right)=\left|F_{\text {out }}\right|\left(-r_{\text {in }}+\frac{1}{r_{\text {in }}^{n-1}}\left(\sqrt{n}\left(l(h)+\frac{d}{2}-r_{1}\right)\right)^{n}\right) \\
& =\left|F_{\text {out }}\right|\left(-r_{\text {in }}+\frac{1}{r_{\text {in }}^{n-1}}\left(\sqrt{n}\left(C_{0} h^{-1 / n}+r_{1}+\frac{d}{2}\right)\right)^{n}\right)
\end{aligned}
$$

and, at the same time,

$$
\left|F_{\text {out }}\right| \geqslant 2 C_{1} \frac{h}{d^{2}} \geqslant 2\left\|\Delta v_{\text {glue }}\right\|_{\infty}
$$

Putting the two together, and using the fact that $F_{\text {in }}$ and $r_{\text {in }}$ are now fixed, it is sufficient to choose $d$ and $h$ so that

$$
C^{\prime}>C \frac{h}{d^{2}}\left(h^{-1 / n}+\frac{d}{2}+r_{1}\right)^{n} .
$$

Since $C \frac{h}{d^{2}}\left(h^{-1 / n}+\frac{d}{2}+r_{1}\right)^{n}<2^{n} C\left(\frac{1}{d^{2}}+\frac{h}{d^{2}}\left(\frac{d}{2}+r_{1}\right)^{n}\right)$, one can see that such a choice is possible. Now we fix $F_{\text {out }}=-2 C_{1} h / d^{2}$.

Now we choose, according to Proposition 2.8, the index set $I$ of relevant obstacles. From Proposition 2.11 and the Lipschitz condition on the percolating cluster of selected boxes from Proposition 2.8, we know there exists a function $v_{\text {glue }}$ whose derivative is only supported on the set $\tilde{D}$ and which has the property $v_{\text {glue }}\left(x_{k}\right)=y_{k}+r_{0}$ for all $k \in I$, and $\left\|\Delta v_{\text {glue }}\right\|_{\infty} \leqslant C_{1} h / d^{2}$.

Choosing $0<F^{*} \leqslant \min \left\{-F_{\text {out }} / 2, \bar{f} / 2\right\}$ one can now see that the function

$$
v=v_{\text {flat }}+v_{\text {glue }}
$$

satisfies

$$
0 \geqslant \Delta v(x)+\sum_{i \in I} \bar{f} \phi\left(x-x_{i}, v(x)-y_{i}\right)+F^{*} \geqslant \Delta v(x)+f(x, v(x), \omega)+F^{*} .
$$


REMARK 2.12 For a slightly different model, if the pinning sites are centered on a regular lattice, i.e., $f(x, y, \omega)=\sum_{i \in \mathbb{Z}^{n}, j \in \mathbb{Z}+1 / 2} f_{i, j}(\omega) \phi(x-i, y-j)$, there is a lower bound for $h-$ one cannot make the boxes more shallow than the lattice spacing. This leads to the fact that there might not exist a $d$ satisfying the estimate (2.5). For $n=1$, one can still find the supersolution in the described way, since the scaling of the gradient of $v_{\text {out }}$ with the distance $d$ works favorably. In particular, the construction works for the model used in [CD].

For $n \geqslant 2$, the construction only works for $\bar{f}$ sufficiently large. Depending on distribution of $f_{1}$, such a choice for $\bar{f}$ might not be possible.

We now turn towards the construction of a supersolution for the mean curvature flow. The theorem itself remains unchanged.

THEOREM 2.13 (Existence of a pinned solution for MCF) If Conditions 2.1 and 2.2 are satisfied, then there exists $F^{*}>0$ and a nonnegative $w: \mathbb{R}^{n} \times \Omega \rightarrow[0, \infty)$ so that a.s., $0 \geqslant \kappa(w(x, \omega))+$ $f(x, w(x, \omega))+F^{*}$, i.e., any solution to (1.1) with initial condition (1.2) and $F \leqslant F^{*}$ gets pinned.

The idea of the proof is to construct a local solution and a gluing function for the mean curvature operator and then provide estimates akin to Propositions 2.6 and 2.11 for these functions. The rest of the proof, modulo an estimate for the behavior of the nonlinear mean curvature operator when adding the local solution and the gluing function, remain unchanged.

Definition 2.14 (Local solution for MCF) As in Definition 2.5, fix $r_{\text {in }} \in\left(0, r_{0}\right), r_{\text {out }}>0$, $F_{\text {in }}>0$, and $F_{\text {out }}<0$, but now making sure that $r_{\text {in }} \leqslant F_{\text {in }}$ and $\left|F_{\text {out }}\right|$ is sufficiently small so that $\frac{\left(r_{\text {out }}-r_{\text {in }}\right)^{n-1}}{r_{\text {out }}^{n}-\left(r_{\text {out }}-r_{\text {in }}\right)^{n}}>\left|F_{\text {out }}\right|$. We construct the local solution from rotationally symmetric surfaces of constant mean curvature, so called Delaunay surfaces [De].

Let $w_{\text {in }}: B_{r_{\text {in }}} \rightarrow \mathbb{R}$ be given as $w_{\text {in }}(x)=-\sqrt{F_{\text {in }}^{2}-|x|^{2}}+\sqrt{F_{\text {in }}^{2}-r_{\text {in }}^{2}}$. The radially symmetric function $w_{\text {out }}: B_{r_{\text {out }}} \backslash B_{r_{\text {in }}} \rightarrow \mathbb{R}$ is defined by an elliptic integral as

$$
w_{\text {out }}(r)=\int_{0}^{r_{\text {out }}-r} \frac{-1}{\sqrt{\frac{\left(r_{\text {out }}-\rho\right)^{2 n-2}}{\left(r_{\text {out }}^{n}-\left(r_{\text {out }}-\rho\right)^{n}\right)^{2} F_{\text {out }}^{2}}-1}} \mathrm{~d} \rho-C,
$$

where $C=\int_{0}^{r_{\text {out }}-r_{\text {in }}} \frac{-1}{\sqrt{\frac{\left(r_{\text {out }}-\rho\right)^{2 n-2}}{\left(r_{\text {out }}^{n}-\left(r_{\text {out }}-\rho\right)^{n}\right)^{2} F_{\text {out }}^{2}}-1}} \mathrm{~d} \rho$.

We define

$$
w_{\text {local }}:= \begin{cases}w_{\text {in }} & \text { on } B_{r_{\text {in }}}, \\ w_{\text {out }} & \text { on } B_{r_{\text {out }}} \backslash B_{r_{\text {in }}}, \\ \lim _{r \rightarrow r_{\text {out }}} w_{\text {out }}(r) & \text { on } \partial B_{r_{\text {out }}}, \\ \infty & \text { otherwise. }\end{cases}
$$

PROPOSITION 2.15 The function $w_{\text {local }}$ is finite on $B_{r_{\text {out }}}$. Furthermore,

$$
0 \geqslant \kappa\left(w_{\text {local }}\right)+\bar{f} \phi\left(\cdot, w_{\text {local }}(\cdot)+r_{0}\right)+F \quad \text { on } B_{r_{\text {out }}}
$$

in the sense of viscosity solutions if 
(i) $0 \geqslant F_{\text {in }}-\bar{f}+F$,

(ii) $0 \geqslant F_{\text {out }}+F$,

(iii) $F_{\text {in }}-\sqrt{F_{\text {in }}^{2}-r_{\text {in }}^{2}} \leqslant r_{0}$.

(iv) $\frac{1}{\sqrt{\frac{\left(r_{\text {out }}-r_{\text {in }}\right)^{2 n-2}}{\left(r_{\text {out }}^{n}-\left(r_{\text {out }}-r_{\text {in }}\right)^{n}\right)^{2} F_{\text {out }}^{2}}-1}}<\frac{r_{\text {in }}}{\sqrt{F_{\text {in }}^{2}-r_{\text {in }}^{2}}}$.

Proof. The first statement is clear by inspection, since under the conditions on $r_{\text {in }}, F_{\text {in }}, r_{\text {out }}$, and $F_{\text {out }}$ the functions $w_{\text {in }}$ and $w_{\text {out }}$ remain finite. The second statement holds due to (i) and (ii) on the inside of the sphere $B_{r_{\text {in }}}$ (condition (iii) confines the graph of $w_{\text {in }}$ to the set where $\phi \leqslant-1$ ) and on the inside of the annulus $B_{r_{\text {out }}} \backslash B_{r_{\text {in }}}$. Condition (iv) ensures that the derivative of $w_{\text {local }}$ jumps only downwards going radially across the boundary from the sphere to the annulus, so that the mean curvature of $w_{\text {local }}$ at the boundary is negative in the viscosity sense.

Proof of Theorem 2.13. In order to employ the construction from the proof of Theorem 2.4, we first need to make sure that the scaling of

$$
\left|\partial_{r} w_{\text {out }}(r)\right|_{r=r_{\text {in }}} \mid=\frac{1}{\sqrt{\frac{\left(r_{\text {out }}-r_{\text {in }}\right)^{2 n-2}}{\left(r_{\text {out }}^{n}-\left(r_{\text {out }}-r_{\text {in }}\right)^{n}\right)^{2} F_{\text {out }}^{2}}-1}}
$$

is suitable. Consider thus $F_{\text {out }}=-c \frac{r_{\text {in }}^{n-1}}{r_{\text {out }}^{n}}$ and note that $\left|\partial_{r} w_{\text {out }}(r)\right|_{r=r_{\text {in }}} \mid$ is then given by

$$
g\left(r_{\mathrm{out}}, c\right):=\frac{1}{\sqrt{\frac{\left(r_{\mathrm{out}}-r_{\mathrm{in}}\right)^{2 n-2} r_{\mathrm{out}}^{2 n}}{\left(r_{\mathrm{out}}^{n}-\left(r_{\mathrm{out}}-r_{\mathrm{in}}\right)^{n}\right)^{2} c^{2} r_{\mathrm{in}}^{2 n-2}}-1}} .
$$

One can see by a simple calculation that for $c$ small enough there exists $C_{2}>0$ so that $g\left(r_{\text {out }}, c\right)<$ $C_{2} c$. This, however, implies that in the correct regime the Delaunay surface from the construction of $w_{\text {out }}$ has the same scaling properties with respect to $r_{\text {out }}$ as the function $v_{\text {out }}$.

The proof of Theorem 2.13 can now be completed by the same construction as for the semilinear equation. First, fix the supersolution inside the obstacles. This determines the maximal outgoing radial derivative $\left.\partial_{r} w_{\text {in }}(r)\right|_{r=r_{\text {in }}}=: G$ and the radius of the inner sphere $r_{\text {in }}$. Consider then the function $w_{\text {flat }}$ constructed analogously to $v_{\text {flat }}$ above. It is necessary to have (after setting $\left.r_{\text {out }}=\sqrt{n}\left(l(h)+d / 2-r_{1}\right)\right)$

$$
g\left(r_{\text {out }}, c\right)<G
$$

as well as

$$
\left|F_{\text {out }}\right|=c \frac{r_{\text {in }}^{n-1}}{\left(\sqrt{n}\left(C_{0} h^{-1 / n}+d / 2-r_{1}\right)\right)^{n}} \geqslant 2 C_{1} \frac{h}{d^{2}} .
$$

The scaling property discussed above ensures that this is possible.

It remains to show that adding the function $v_{\text {glue }}$ from Proposition 2.11 does not destroy the property of negative mean curvature. Define $v(u):=\sqrt{1+|\nabla u|^{2}}$. We have, after collecting terms from expanding the divergence in the mean curvature operator, 


$$
\begin{aligned}
\kappa\left(w_{\text {out }}+v_{\text {glue }}\right)= & \frac{\Delta\left(w_{\text {out }}+v_{\text {glue }}\right)}{v\left(w_{\text {out }}+v_{\text {glue }}\right)}+\frac{\left(\left(D^{2} w_{\text {out }}+D^{2} v_{\text {glue }}\right) \cdot\left(\nabla w_{\text {out }}+\nabla v_{\text {glue }}\right), \nabla w_{\text {out }}+\nabla v_{\text {glue }}\right)}{v\left(w_{\text {out }}+v_{\text {glue }}\right)^{3}} \\
= & \kappa\left(w_{\text {out }}\right)+\frac{\Delta v_{\text {glue }}}{v\left(w_{\text {out }}+v_{\text {glue }}\right)}+\Delta w_{\text {out }}\left(\frac{1}{v\left(w_{\text {out }}+v_{\text {glue }}\right)}-\frac{1}{v\left(w_{\text {out }}\right)}\right) \\
& +\left(D^{2} w_{\text {out }} \cdot \nabla w_{\text {out }}, \nabla w_{\text {out }}\right)\left(\frac{1}{v\left(w_{\text {out }}+v_{\text {glue }}\right)^{3}}-\frac{1}{v\left(w_{\text {out }}\right)^{3}}\right) \\
& +\frac{\left(D^{2} v_{\text {glue }} \cdot\left(\nabla w_{\text {out }}+\nabla v_{\text {glue }}\right), \nabla w_{\text {out }}+\nabla v_{\text {glue }}\right)}{v\left(w_{\text {out }}+v_{\text {glue }}\right)^{3}} \\
& +\frac{2\left(D^{2} w_{\text {out }} \cdot \nabla w_{\text {out }}, \nabla v_{\text {glue }}\right)}{v\left(w_{\text {out }}+v_{\text {glue }}\right)^{3}}+\frac{\left(D^{2} w_{\text {out }} \cdot \nabla v_{\text {glue }}, \nabla v_{\text {glue }}\right)}{v\left(w_{\text {out }}+v_{\text {glue }}\right)^{3}} \\
= & \kappa\left(w_{\text {out }}\right)+\mathcal{O}\left(\left\|\Delta v_{\text {glue }}\right\|_{\infty}\right)+\mathcal{O}\left(\left\|\Delta w_{\text {out }}\right\|_{\infty}\left\|\nabla w_{\text {out }}\right\|_{\infty}\left\|\nabla v_{\text {glue }}\right\|_{\infty}\right) \\
& +\mathcal{O}\left(\left\|D^{2} w_{\text {out }}\right\|_{\infty}\left\|\nabla w_{\text {out }}\right\|_{\infty}^{2}\left\|\nabla w_{\text {out }}\right\|_{\infty}\left\|\nabla v_{\text {glue }}\right\|_{\infty}\right) \\
& +\mathcal{O}\left(\left\|D^{2} v_{\text {glue }}\right\|_{\infty}\left(\left\|\nabla w_{\text {out }}\right\|_{\infty}^{2}+\left\|\nabla v_{\text {glue }}\right\|_{\infty}^{2}\right)\right. \\
& +\mathcal{O}\left(\left\|D^{2} w_{\text {out }}\right\|_{\infty}\left(\left\|\nabla v_{\text {glue }}\right\|_{\infty}\left\|\nabla w_{\text {out }}\right\|_{\infty}+\left\|\nabla v_{\text {glue }}\right\|_{\infty}^{2}\right) .\right.
\end{aligned}
$$

Here, $(\cdot, \cdot)$ denotes the scalar product in $\mathbb{R}^{n}$, and $D^{2} v \cdot \nabla w$ denotes the matrix-vector product of the matrix of second derivatives of $v: \mathbb{R}^{n} \rightarrow \mathbb{R}$ applied to the gradient vector of $w: \mathbb{R}^{n} \rightarrow \mathbb{R}$. We have used the $\mathcal{O}$-Landau notation to denote the leading order behavior of terms for small argument. Note that $\left\|\nabla v_{\text {glue }}\right\|_{\infty}=\mathcal{O}(h / d),\left\|D^{2} v_{\text {glue }}\right\|_{\infty}=\mathcal{O}\left(h / d^{2}\right)$ (Proposition 2.11), and $\left\|\nabla w_{\text {out }}\right\|_{\infty}=\mathcal{O}(c)$. One can see that also $\left\|D^{2} w_{\text {out }}\right\|_{\infty}=\mathcal{O}(c)$, since for small curvature the gradient term dominates when calculating the second derivatives of the function $v_{\text {out }}$. All error terms can be made small with respect to

$$
\left|\kappa\left(w_{\text {out }}\right)\right|=\left|F_{\text {out }}\right|=c \frac{r_{\text {in }}^{n-1}}{\left(\sqrt{n}\left(C_{0} h^{-1 / n}+d / 2-r_{1}\right)\right)^{n}},
$$

by noting that one can, instead of (2.7), for a given $C>0$, fix $h$ and $d$ so that $-F_{\text {out }}>C h / d$. The rest of the proof then follows that of Theorem 2.4.

\section{Conclusions}

We have shown that, for our models of interface evolution in random media, a finite critical force is required to propagate the interface through the body. Many questions in this area, however, remain open. It was shown in [CD] that for a model with obstacles on lattice sites ${ }^{3}$ with random exponentially distributed strength for $n=1$, no more stationary solution can exist if the forcing exceeds a critical value. The question whether interfaces in this case move with a finite speed of propagation is still open and currently under investigation (this is of course trivial for uniformly bounded obstacle strength when also avoiding overlap of obstacles). Pinning for small $F$ and depinning for larger $F$ imply that there is a transition from a viscous kinetic relation (i.e., velocity $=$ force) in the microscopic model (after a time-rescaling) to a rate independent model for the macroscopic behavior of the system. Such rate independent kinetics are commonly assumed

\footnotetext{
3 As pointed out in Remark 2.12, our construction of a supersolution for sufficiently small external driving force also works in this "lattice case" for $n=1$.
} 
in macroscopic models of phase transformations or plasticity. This article provides a step to deriving this assumption from microscopic viscous kinetics.

\section{Acknowledgements}

P. Dondl and M. Scheutzow acknowledge support from the DFG-funded research group 'Analysis and Stochastics in Complex Physical Systems' (FOR 718).

\section{REFERENCES}

[B] BrazovskiI, S., \& Nattermann, T. Pinning and sliding of driven elastic systems: from domain walls to charge density waves. Adv. Phys. $\mathbf{5 3}$ (2004), 177-252.

[CL] Cardaliaguet, P., Lions P.-L., \& Souganidis, P. E. A discussion about the homogenization of moving interfaces. J. Math. Pures Appl. 91 (2009), 339-363. Zbl 1180.35070 MR 2518002

[CD] COVILlE, J., DiRR, N., \& LuCKHAUS, S. Non-existence of positive stationary solutions for a class of semi-linear PDEs with random coefficients. Networks Heterogeneous Media 5 (2010), 745-763. MR 2740531

[CB] Craciun, B., \& Bhattacharya, K. Effective motion of a curvature-sensitive interface through a heterogeneous medium. Interfaces Free Bound. 6 (2004), 151-173. Zbl 1061.35148 MR 2079601

[De] Delaunay, C. Sur la surface de révolution dont la courbure moyenne est constante. J. Math. Pures Appl. 1 (1841), 309-320.

[DD] Dirr, N., Dondl, P. W., Grimmett, G. R., Holroyd, A. E., \& Scheutzow, M. Lipschitz percolation. Electron. Comm. Probab. 15 (2010), 14-21. Zbl 1193.60115 MR 2581044

[DK] DirR, N., Karali, G., \& YIP, N. K. Pulsating wave for mean curvature flow in inhomogeneous medium. Eur. J. Appl. Math. 19 (2008), 661-699. Zbl 1185.53076 MR 2463225

[DY] DIRR, N., \& YIP, N. K. Pinning and de-pinning phenomena in front propagation in heterogeneous media. Interfaces Free Bound. 8 (2006), 79-109. Z Zbl 1101.35074 MR 2231253

[G] Grimmett, G. R., \& Holroyd, A. E. Geometry of Lipschitz percolation. Ann. Inst. H. Poincaré Probab. Statist., to appear.

[Ka] Kardar, M. Nonequilibrium dynamics of interfaces and lines. Phys. Rep. 301 (1998), 85-112.

[K1] KLeEmanN, W. Dynamic phase transitions in ferroic systems with pinned domain wall. Oberwolfach Reports 1 (2004), 1587-1656. 